\title{
(6) OPEN ACCESS \\ A profile of suspected child abuse as a subgroup of major trauma patients
}

\author{
Ffion C Davies, ${ }^{1}$ Timothy J Coats, ${ }^{2}$ Ross Fisher, ${ }^{3}$ Thomas Lawrence, ${ }^{4}$ Fiona E Lecky ${ }^{5}$
}

${ }^{1}$ Emergency Department, University Hospitals of Leicester NHS Trust, Leicester Royal Infirmary, Leicester, UK ${ }^{2}$ Department of Emergency Medicine, University of Leicester, Leicester, UK ${ }^{3}$ Department of Surgery, Sheffield Children's Hospital, Sheffield, UK

${ }^{4}$ Trauma Audit Research Network, Salford Royal NHS Foundation Trust, Salford, UK ${ }^{5}$ Health Services Research, School of Health and Related Research (ScHARR), University of Sheffield, Sheffield, UK

\section{Correspondence to} Dr Ffion C Davies, Emergency Department, University Hospitals of Leicester NHS Trust, Leicester Royal Infirmary, Leicester LE1 5WW, UK; Ffion.davies@uhl-tr.nhs.uk

Received 4 August 2015 Revised 20 October 2015 Accepted 24 October 2015

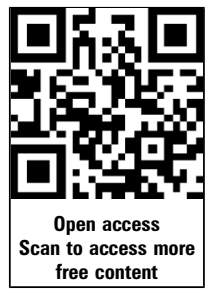

CrossMark

\section{To cite: Davies FC,} Coats TJ, Fisher $\mathrm{R}$, et al. Emerg Med J 2015;32: 921-925.

\section{ABSTRACT}

Introduction Non-accidental injury (NAI) in children is an important cause of major injury. The Trauma Audit Research Network (TARN) recently analysed data on the demographics of paediatric trauma and highlighted NAI as a major cause of death and severe injury in children. This paper examined TARN data to characterise accidental versus abusive cases of major injury. Methods The national trauma registry of England and Wales (TARN) database was interrogated for the classification of mechanism of injury in children by intent, from January 2004 to December 2013. Contributing hospitals' submissions were classified into accidental injury (AI), suspected child abuse (SCA) or alleged assault (AA) to enable demographic and injury comparisons.

Results In the study population of 14845 children, 13708 (92.3\%, Cl $91.9 \%$ to $92.8 \%)$ were classified as accidental injury, 368 as alleged assault $(2.5 \%, \mathrm{Cl} 2.2 \%$ to $2.7 \%$ ) and 769 as SCA $(5.2 \%, \mathrm{Cl} 4.8 \%$ to $5.5 \%)$. Nearly all cases of severely injured children suffering trauma because of SCA occurred in the age group of 0 5 years (751 of $769,97.7 \%$ ), with $76.3 \%$ occurring in infants under the age of 1 year. Compared with accidental injury, suspected victims of abuse have higher overall injury severity scores, have a higher proportion of head injury and a threefold higher mortality rate of $7.6 \%(\mathrm{Cl} 5.51 \%$ to $9.68 \%)$ vs $2.6 \%(\mathrm{Cl} 2.3 \%$ to $2.9 \%)$.

Conclusions This study highlights that major injury occurring as a result of SCA has a typical demographic pattern. These children tend to be under 12 months of age, with more severe injury. Understanding these demographics could help receiving hospitals identify children with major injuries resulting from abuse and ensure swift transfer to specialist care.

\section{INTRODUCTION}

Children account for around 10\% of major trauma in developed world countries and are known to present to the major trauma system in a different way from adults. ${ }^{12}$ The creation of Major Trauma Networks over the last 5 years has radically changed the delivery of trauma care in the UK, and preliminary data are very encouraging in showing that patient outcomes have improved. ${ }^{3}$ These changes were made on evidence of improved outcome in adults, with little data available on the influence of organised trauma systems on outcomes in paediatric major trauma.

Within the UK, national trauma audit is performed by the Trauma Audit Research Network (TARN), which includes data from $96 \%$ of acute hospitals in England and Wales, and is funded by

\section{Key messages}

What is already known on this subject?

- Non-accidental injury (NAI) is known to be an important cause of major trauma in small children (reference 1 in paper, Trauma Audit Research Network (TARN) 2012 report on Severe Injury in Children, reference 2 from the Victoria Trauma Registry, reference 6 from Israel).

- Patients injured as a result of NAI are likely to have severe brain injury (reference 5).

- No previous study has compared the demography, injuries and outcome of NAI versus non-NAl as a mechanism of injury in severely injured children.

\section{What might this study add?}

- This study compares NAI with accidental injury and assault not due to NAI, for the UK paediatric population entered into the TARN database, for the 10-year period 2004-2013.

- NAl as a mechanism of injury is associated with age under 1, higher injury severity score, severe brain injury and higher mortality rates than children accidentally injured. These findings may have implications for the design of major trauma networks.

individual hospital membership subscriptions. The TARN report 'Severe Injury in Children 2012'1 identified non-accidental injury (NAI) as a significant cause of injury in the under 2 -year olds. This led us to examine whether abused children form a distinct subset with different characteristics compared with children who are accidentally injured.

Children severely injured as a result of abuse are under-researched, with the published literature around child abuse often focussing on minor injuries (as markers to allow early detection of NAI before major injury) rather than major injury. In contrast, media reports and national inquiries are focused on deaths, so non-fatal major injury is relatively poorly described. There has been no previous national overview of severe injuries caused by nonaccidental injury. We sought to address this by characterising the demographic and injury characteristics of children who sustained abusive major trauma compared with unintentional injury.

\section{METHODS}

The TARN database from January 2004 to December 2013 was examined. Hospitals report 
patients to this database if they suffer injury and are either admitted to hospital for at least 3 days, admitted to a critical care area, are transferred for specialist care or die in hospital. Patients who die before reaching hospital (no hospital resuscitation performed) are not included. The number of hospitals contributing data increased over the time of the study from 84 in 2004 to 169 in 2013.

The inclusion criteria for this study were patients under 16 years old at presentation to hospital, with data submitted to TARN, including both those patients admitted directly to hospital and those admitted indirectly (via another hospital) in England and Wales.

TARN data coordinators (specifically trained clerical staff or nurses) in submitting hospitals enter 'intent' as a data category. There are seven categories. These categories enabled study patients to be divided into three groups according to whether there was accidental injury (AI) or whether the injury was categorised as from alleged assault (AA) or suspected child abuse (SCA).

Data entry staff enter SCA as a category of intent, only when there is clear evidence in the hospital notes that the clinical staff were investigating child abuse with a high degree of suspicion. It is not possible for data entry staff to know at that stage whether the ultimate findings were conclusive as to the likelihood of abuse. In our analysis, only cases entered as SCA to the database were included in the SCA group.

Alleged assault is a category of intent within TARN. All of these cases were attributed to the AA group in our analysis. The database includes descriptors, enabling AA cases to be examined in more detail, and showed that this category commonly includes mechanisms of injury such as adolescents fighting. AA cases may have included potential child abuse, but as the cases were indeterminate, it is not possible to ascribe these to the SCA category.

Cases attributed to accidental injury were derived from the remaining five options for categorising intent of injury. These categories are non-intentional, sport, intent inconclusive, suspected high-risk behaviour and alleged intent (non-assault).
Children in the category 'alleged intent (non-assault)' were few in number, and examination of their data implied that this was play rather than abuse. All of these other categories were, therefore, classed as AI in this study.

Children classified into the three categories of injury intent (AI, AA and SCA) were compared in terms of age, gender, injury severity, body regions injured and mortality.

Mortality is recorded at discharge from hospital; however, in some instances where patients are transferred between hospitals, TARN data may not be complete and it may not be possible to determine the final outcome due to missing data. These patients are detailed in figure 1.

Demographic and injury data have been categorised and reported as number (percentage). Pearson's $\chi^{2}$ test was used to test the distribution and significance of these variables. Mortality is reported as a percentage value and 95\% CIs were calculated around these figures. $\mathrm{p}$ Values are derived from Pearson's $\chi^{2}$ test. Data extraction and analysis was performed using SQL Server 2012, Excel (Microsoft, Redmond, California, USA) and STATA (StataCorp, College Station, Texas, USA).

TARN has Health Research Authority (PIAGG Section 20) approval to conduct research on anonymised data.

\section{RESULTS}

There were 15936 children recorded in the TARN database during the 10-year period from January 2004 to December 2013. Of these, $981(6.2 \%)$ were excluded as there was no record of 'intent', a further $110(0.7 \%)$ were removed where the intent was documented as self-harm, leaving a study population of 14845 cases. All data presented are on this population of 14845 , apart from the mortality analysis (12 511 cases), for which 2334 cases with unknown outcome were excluded.

The study population was attributed to one of three categories: AI, AA and SCA. AI accounted for 13708 (92.3\%, CI $91.9 \%$ to $92.8 \%)$ of cases, AA for $368(2.5 \%$, CI $2.2 \%$ to $2.7 \%$ ) of cases and 769 children $(5.2 \%$, CI $4.8 \%$ to $5.5 \%)$ had injuries attributed to SCA (see table 1).

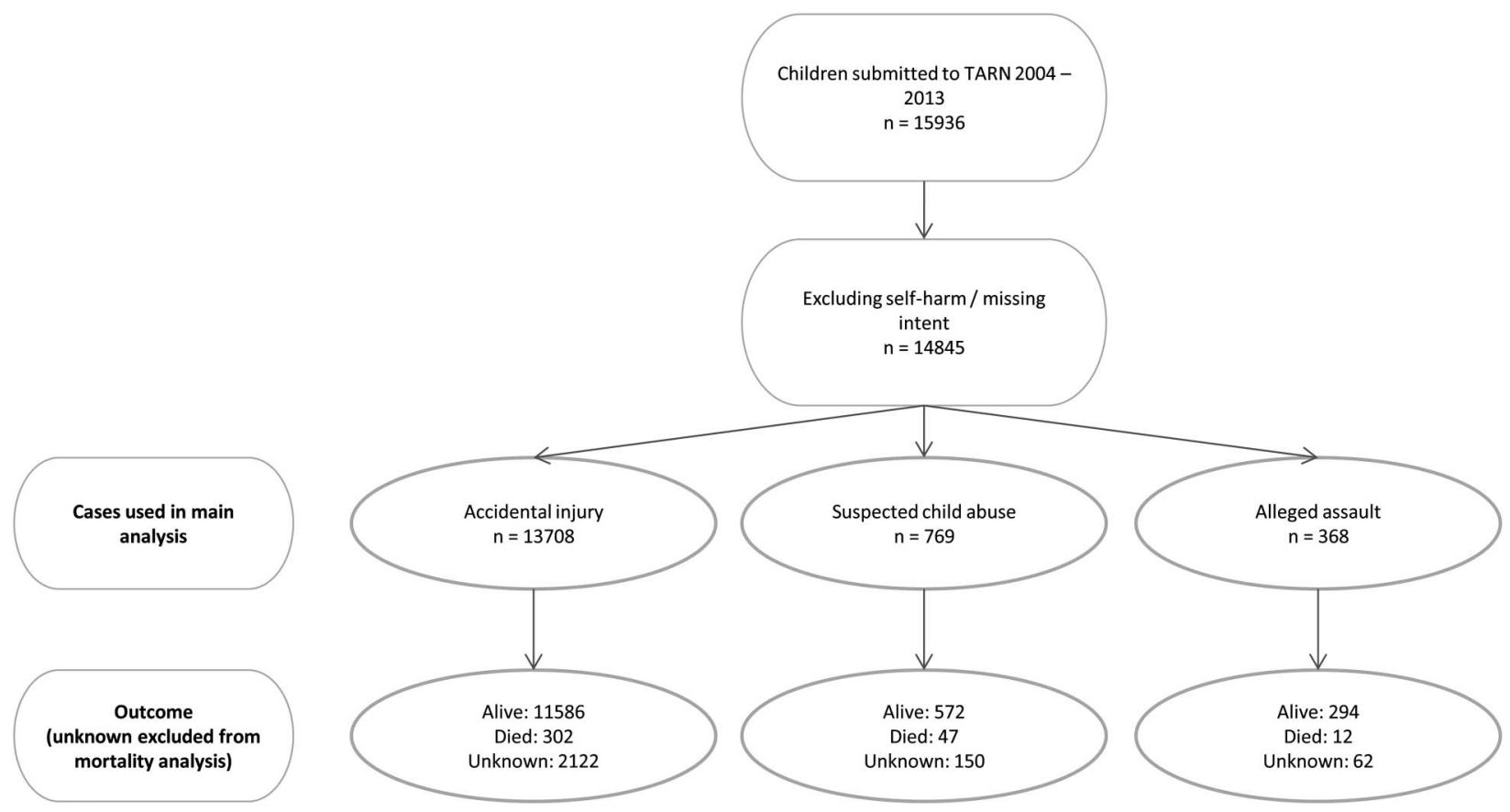

Figure 1 Case identification. TARN, Trauma Audit Research Network. 
Table 1 Profile of cases by injury intent

\begin{tabular}{|c|c|c|c|c|c|}
\hline Category & Group & $\begin{array}{l}\text { Accidental injury } \\
\text { n (\%) }\end{array}$ & $\begin{array}{l}\text { Alleged assault } \\
\text { n (\%) }\end{array}$ & $\begin{array}{l}\text { Suspected child abuse } \\
\text { n (\%) }\end{array}$ & p Value* \\
\hline $\mathrm{n}$ & & 13708 & 368 & 769 & \\
\hline Age & $\begin{array}{l}\text { Under } 1 \\
1-4 \\
5-10 \\
11-15\end{array}$ & $\begin{array}{l}1296(9.5) \\
3617(26.4) \\
3670(26.8) \\
5125(37.4)\end{array}$ & $\begin{array}{c}13(3.5) \\
15(4.1) \\
29(7.9) \\
311(84.5)\end{array}$ & $\begin{array}{c}587(76.3) \\
164(21.3) \\
16(2.1) \\
2(0.3)\end{array}$ & $<0.001$ \\
\hline Gender & $\begin{array}{l}\text { Female } \\
\text { Male }\end{array}$ & $\begin{array}{l}4290(31.3) \\
9418(68.7)\end{array}$ & $\begin{array}{r}39(10.6) \\
329(89.4)\end{array}$ & $\begin{array}{l}315(41) \\
454(59)\end{array}$ & $<0.001$ \\
\hline ISS & $\begin{array}{l}1-8 \\
9-15 \\
>15\end{array}$ & $\begin{array}{l}1671(12.2) \\
7520(54.9) \\
4517(33)\end{array}$ & $\begin{array}{l}79(21.5) \\
164(44.6) \\
125(34)\end{array}$ & $\begin{array}{l}101(13.1) \\
226(29.4) \\
442(57.5)\end{array}$ & $<0.001$ \\
\hline AIS 3+ injury & $\begin{array}{l}\text { Head } \\
\text { Torso } \\
\text { Limbs } \\
\text { Other } \\
\text { No 3+ injury }\end{array}$ & $\begin{array}{c}4302(31.4) \\
2137(15.6) \\
6658(48.6) \\
519(3.8) \\
1746(12.7)\end{array}$ & $\begin{array}{l}112(30.4) \\
164(44.6) \\
29(7.9) \\
10(2.7) \\
79(21.5)\end{array}$ & $\begin{aligned} 441 & (57.3) \\
77 & (10) \\
204 & (26.5) \\
39 & (5.1) \\
107 & (13.9)\end{aligned}$ & $\begin{array}{l}<0.001 \\
<0.001 \\
<0.001 \\
<0.001 \\
<0.001\end{array}$ \\
\hline Mortality (\%) $(95 \% \mathrm{CI})$ & & 2.61 (2.32 to 2.9$)$ & $3.92(1.75$ to 6.1$)$ & 7.59 (5.51 to 9.68$)$ & \\
\hline
\end{tabular}

The three different categories were profiled for age and gender characteristics. In the AI category, there was a male preponderance (68.7\% male, 95\% CI $67.9 \%$ to $69.5 \%)$, cases of SCA showed a relatively even gender balance $(59.0 \%$ male) and the AA category showed clear gender differences with many more boys suffering injury from alleged assault $(89.4 \%$ male, 95\% CI $86.3 \%$ to $92.5 \%$ ).

Examining the age distribution, a very clear pattern was seen when the 'intent' was examined across different age bands (table 1). Of all SCA cases in the TARN database for this period, nearly all cases $(97.7 \%$, 95\% CI $96.6 \%$ to $98.8 \%$ ) occurred in children under 5 years of age. Also, 587 of the 769 cases $(76.3 \%, 95 \%$ CI $73.3 \%$ to $79.3 \%)$ occurred in children under the age of $1,21.3 \%$ (95\% CI $18.4 \%$ to $24.2 \%$ ) in the age group of $1-4$ years and only $2.4 \%$ (95\% CI $1.3 \%$ to $3.5 \%$ ) of cases were over the age of five.

The age band data are displayed in figure 2, while figure 3 portrays the age profile of SCA across the study population, showing that in the context of severe injury (ie, TARN eligibility) SCA affects mainly children under 1 year of age. The median age of all SCA cases was 3.6 months, IQR 1.210.8 months.
The category of AA was submitted in 368 (2.5\%) of the 14845 study population. Figure 2 shows the age profile of AA, which is the reverse of the age profile of SCA cases. AA was submitted as the mechanism of injury in $0.7 \%$ of under $1 \mathrm{~s}, 0.4 \%$ of $1-4$ year olds, $0.8 \%$ of $5-10$ year olds and $5.7 \%$ of $11-15$ year olds.

Body region of injury was analysed, for injuries with an abbreviated injury score $\geq 3$ (table 1 ). Children in the AA category are more likely to suffer significant torso injury $(44.6 \%$, $95 \%$ CI $39.5 \%$ to $49.7 \%)$ than head or limb injury $(\mathrm{p}<0.001)$. Accidentally injured children are most likely to suffer significant limb injury $(49 \%, 95 \%$ CI $47.7 \%$ to $49.4 \%, p<0.0001)$ and children injured through abuse have significant head injury as the most likely injury $(57.3 \%$, 95\% CI $53.8 \%$ to $60.8 \%$, $\mathrm{p}<0.001)$.

The three categories of intent were then compared for overall injury severity. The injury severity scores (ISS) for the three groups showed that the highest proportion of patients with ISS $>15$ was seen in SCA cases (figure 4), who are 1.7 times more likely to have an ISS $>15$ than children accidentally injured.

The higher ISS scores in the SCA group are reflected by mortality data (figure 5). Figure 1 highlights how outcome data (dead or alive) were not available for 2334 (15.7\%) cases.

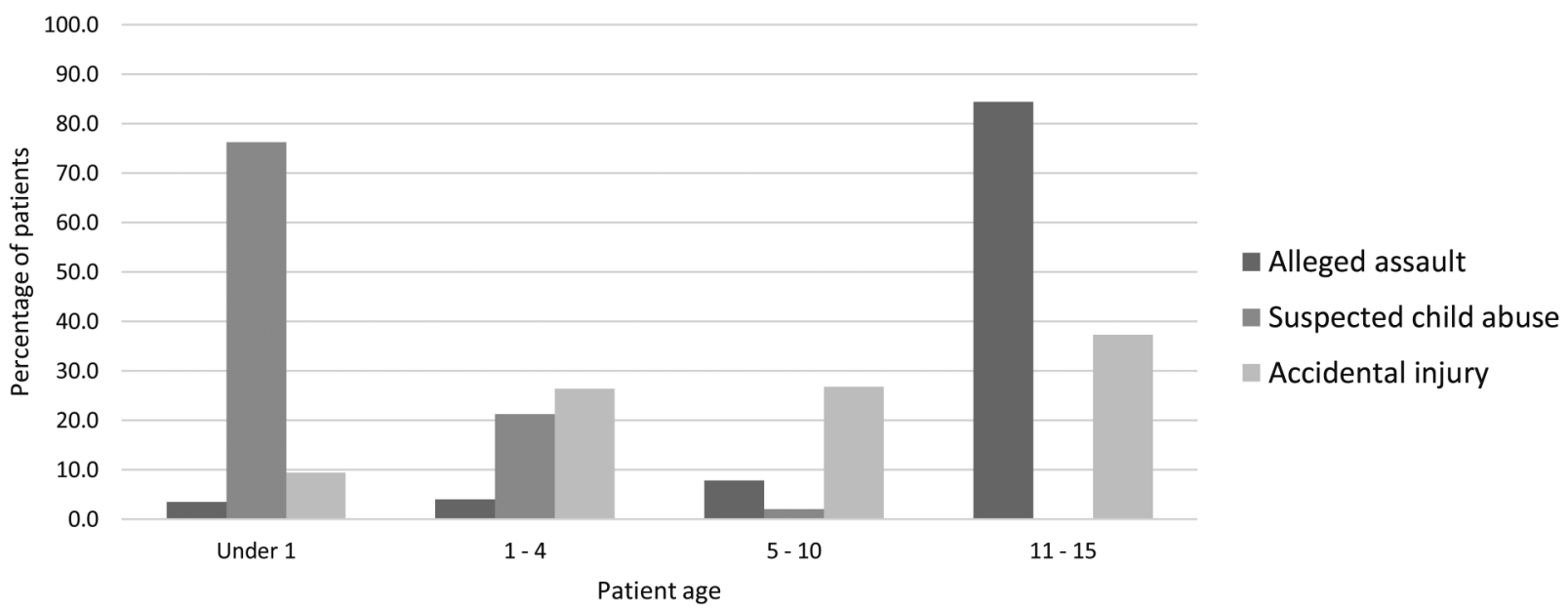

Figure 2 Mechanism of injury by age band. 
Figure 3 Age distribution of suspected child abuse cases.

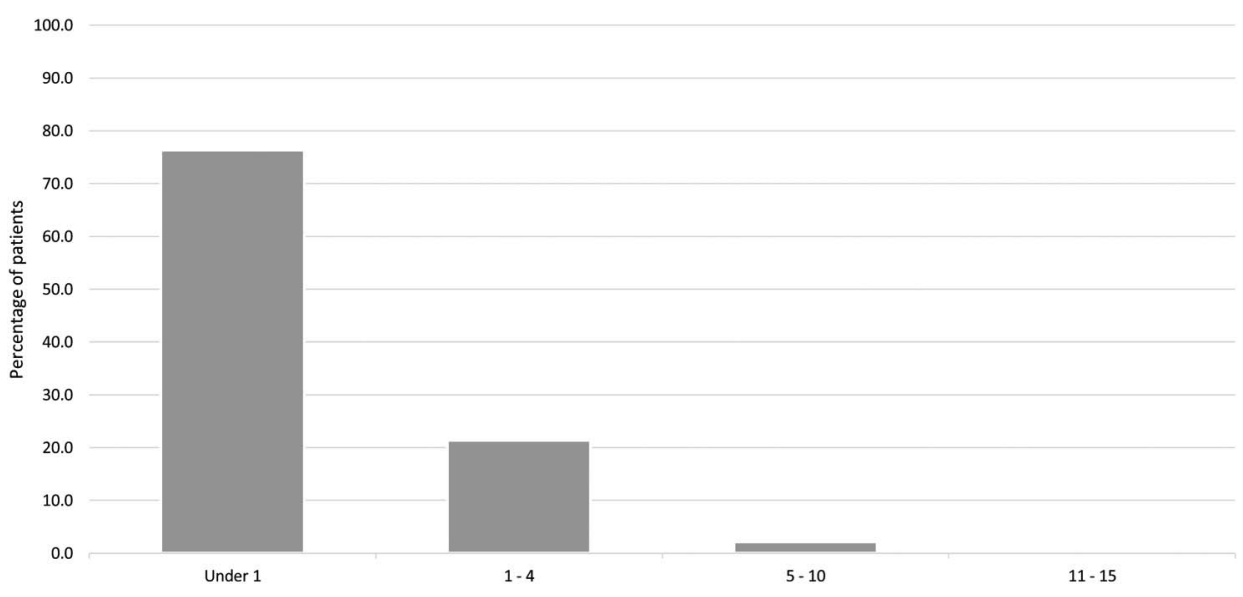

Where outcome was known, it showed a mortality rate of $2.6 \%$ (95\% CI 2.3\% to 2.9\%) following AI (302 deaths out of 11586 children) compared with $7.6 \%$ (95\% CI 5.5\% to 9.7\%) in the SCA category (47 deaths out of 619 children), a nearly threefold higher rate of death than accidental injury. The alleged assault category also had a high mortality rate at 3.9\% (95\% CI 1.7\% to $6.1 \%, 12$ deaths among 306 children) with this group including incidents such as stabbing and other serious assaults.

\section{DISCUSSION}

This study describes SCA over a decade of major trauma data in the UK. This study builds on previous TARN information looking at severe paediatric trauma in a calendar year, ${ }^{1}$ and the new data show that cases of injury from SCA have a different demography than accidentally injured children. They are more likely to sustain a significant head injury, more likely to have an ISS $>15$ and more likely to die than cases of accidental trauma.

The majority of cases of SCA $(76.3 \%)$ were under 1 year of age. This age distribution is similar to that found in an 8 -year analysis of the trauma registry of the state of Victoria, Australia. ${ }^{2}$ In the age group 0-14 years, the incidence of abuse was $3 \%$, with virtually all cases in the age group of $0-4$ years. The TARN 2012 data $^{1}$ showed a bimodal age distribution of severe injury (all causes) in children, with one peak at age under 1 , but the majority of cases occurring between 6 and 15 years of age. An American study of under 5 years old with blunt trauma ${ }^{4}$ revealed a median age of 12.8 months in the abused population. A study of traumatic brain injury $(\mathrm{TBI})^{5}$ showed that TBI secondary to abuse had a median age of 1 year of age versus
8 years in accidental TBI. A review of the Israeli National Trauma Registry in 2009 also identified a peak incidence of severe injury in newborn infants. ${ }^{6}$ So there appears to be a clear pattern of age, in that older children are much less likely to sustain severe injury from abuse than infants. Child abuse is not absent in the age group of 11-15years in the UK, but the TARN major trauma data contained only two cases of SCA in this age group. It may be simply that the more robust physique of an older child means that major trauma is more difficult to inflict.

It is interesting to compare the actual age range for abuse to the high-profile cases of child abuse reported in the media who are often older. This represents a reporting bias that focuses on 'missed opportunities' for intervention and prevention (older children inevitably have more time for recognition and intervention), rather than a true picture of the demography of death and severe injury in non-accidental injury. There is a danger that this reporting bias influences key staff such as social workers, doctors and teachers on older children, when the overwhelming risk of major trauma and death occurs in the first few months of life.

The higher injury severity and mortality rates found for SCA in the TARN data (three times higher than accidental injury) has been seen in other data sets, so has the high rate of severe head injury in this population (57.3\% in our study). Abused children in a study of TBI ${ }^{5}$ had longer intensive care unit stays (mean 3 days vs 1 day), and a 12-year study of an American trauma registry ${ }^{7}$ also demonstrated a median ISS of 10 for cases of abuse compared with 4 for non-abused cases. This same study showed a mortality rate sixfold higher ( $12 \%$ vs $2 \%)$. In another

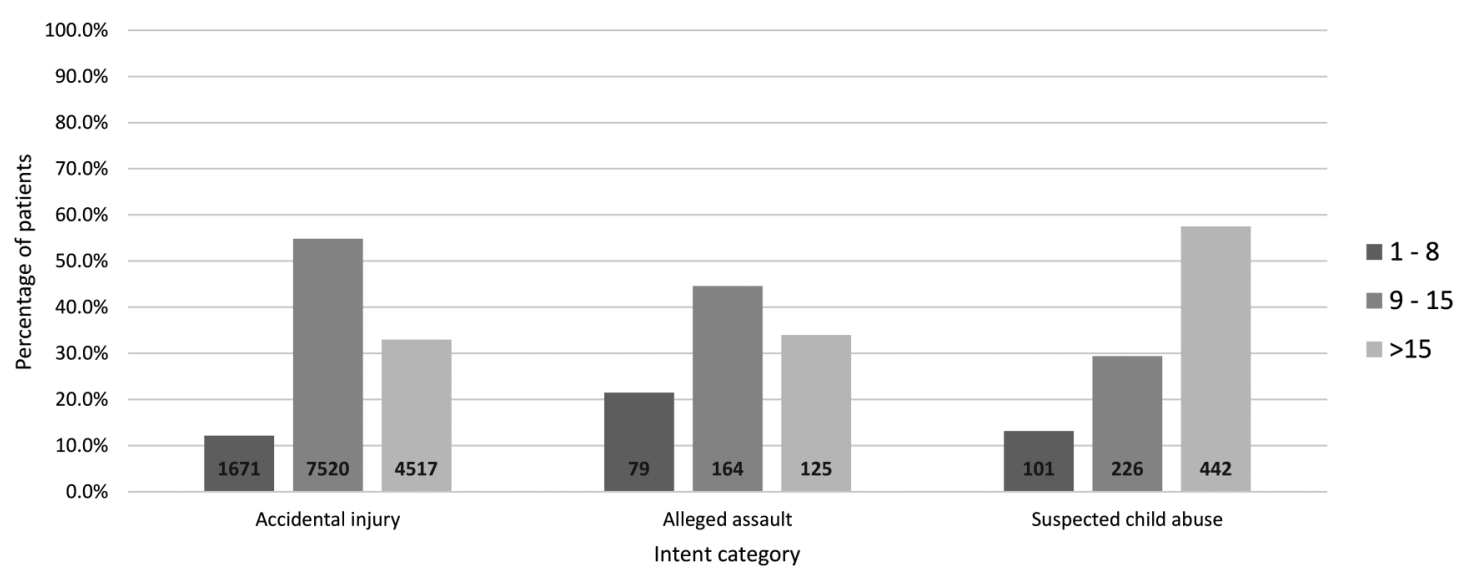

Figure 4 Injury severity score (ISS) range by injury intent. 


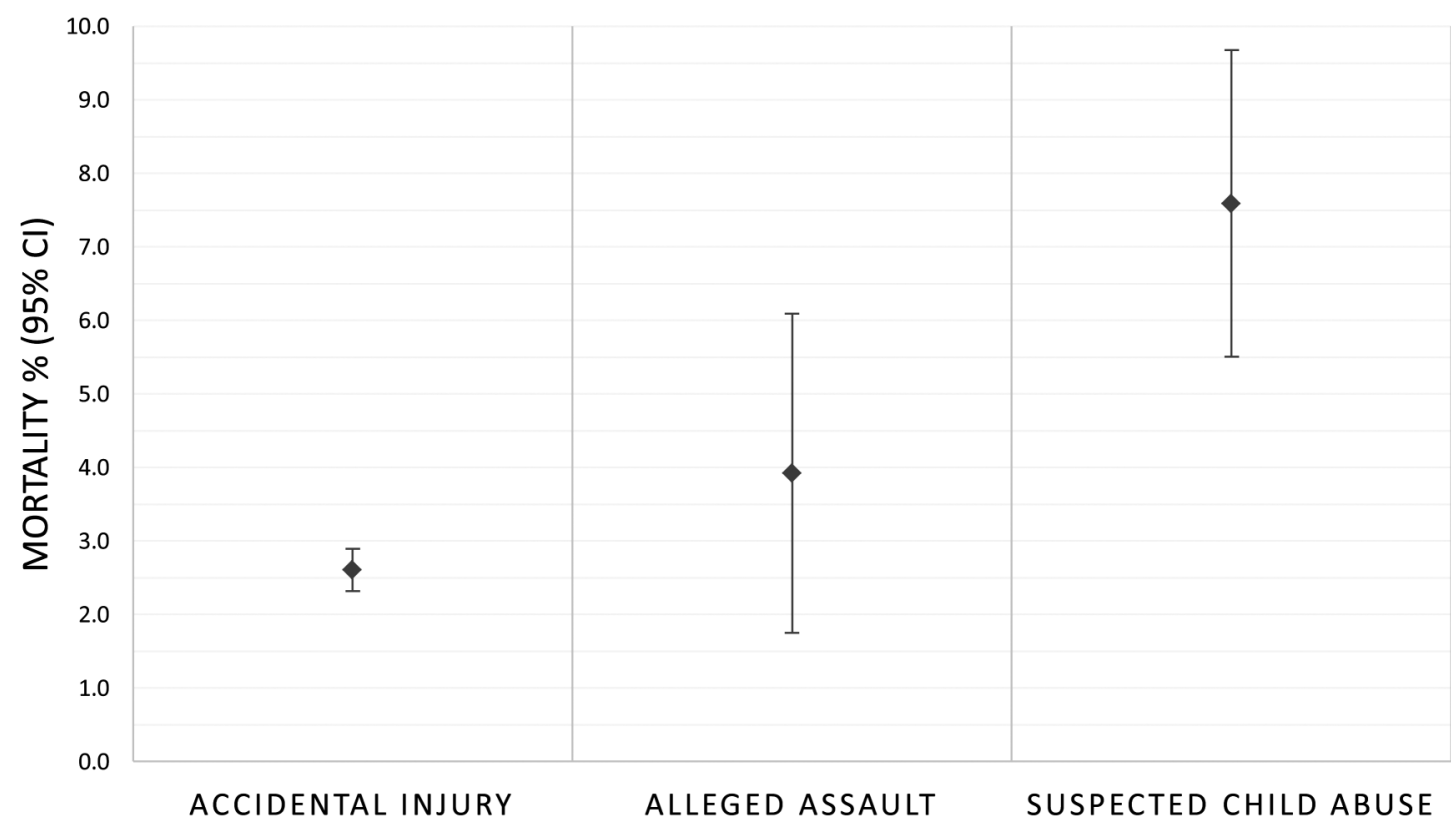

Figure 5 Mortality by injury intent.

study, survival to discharge in a study of children under 5 years was $87.3 \%$ for abused children versus $97.4 \%$ for accidentally injured children. ${ }^{4}$

This study has a number of potential limitations. Data submission to TARN is incomplete, although hospital participation in TARN increased during the decade of the study from 98 hospitals in England and Wales, to 190 (nearly 100\%). The data are submitted by clinical coding staff or nurses, who can only classify 'intent' by what is written in the notes. Interpretation of intent from clinical notes can be difficult. Even experts can find it difficult to determine intent, so a degree of misclassification in the TARN data set seems inevitable. It is likely that SCA will tend to be under-reported as the term is sometimes avoided in clinical notes if there is not significant evidence.

In the age group of $0-4$ years, there were 15 cases that were attributed to alleged assault but not specifically to SCA. From the limited data recorded in the database, these appeared to be sibling or friend-related in the main (such as one toddler pushing another over in a nursery causing a head injury), so the authors do not believe that many of these would have been more accurately categorised as SCA.

\section{CONCLUSION}

This study adds to previous studies of child abuse as a cause of major injury, and in the context of major trauma describes a different demography, pattern of injury and presentation of abusive injury compared with accidental injury. In the TARN database of major trauma, suspected victims of abuse are usually under 1 year old, with a higher ISS mainly due to severe head injury, and have a higher mortality rate than accidentally injured children.

Contributors All authors contributed substantially to the writing.

Competing interests None declared.

Provenance and peer review Not commissioned; externally peer reviewed.

Data sharing statement The data were from the TARN database. Further data are held by TARN and available from the TARN research committee via the TARN website.

Open Access This is an Open Access article distributed in accordance with the Creative Commons Attribution Non Commercial (CC BY-NC 4.0) license, which permits others to distribute, remix, adapt, build upon this work non-commercially, and license their derivative works on different terms, provided the original work is properly cited and the use is non-commercial. See: http://creativecommons.org/ licenses/by-nc/4.0/

\section{REFERENCES}

1 Severe Injury in Children (England \& Wales). Trauma Audit \& Research Network. 2012. http://www.tarn.ac.uk

2 Deasy $C$, Gabbe B, Palmer $C$, et al. Paediatric and adolescent trauma care within an integrated trauma system. Injury 2012;43:2006-11.

3 https://www.tarn.ac.uk/Content.aspx?c=3477

4 Discala C, Siege R, Li G, et al. Child abuse and unintentional injuries: a 10-year retrospective. Arch Pediatr Adolesc Med 2000;154/1:1072-4710.

5 Deans $K$, Minneci $P$, Lowell W, et al. Increased morbidity and mortality of traumatic brain injury in victims of non-accidental trauma. J Trauma Acute Care Surg 2013;75:157-60.

6 Rozenfeld M, Peleg K. Violence-related injury of children in Israel: age-dependent pattern. Bull World Health Organ 2009;87/5:362-8.

7 Chang D, Knight V, Ziegfeld S, et al. The tip of the iceberg for child abuse: the critical roles of the pediatric trauma service and its registry. I Trauma 2004;57:1189-98. 